\title{
TEST SETS FOR NONNEGATIVITY OF POLYNOMIALS INVARIANT UNDER A FINITE REFLECTION GROUP
}

\author{
by \\ José Gabriel Acevedo Habeych
}

Thesis advisor

Ph.D Mauricio Velasco Gregory

A thesis presented for the degree of

Master in Mathematics

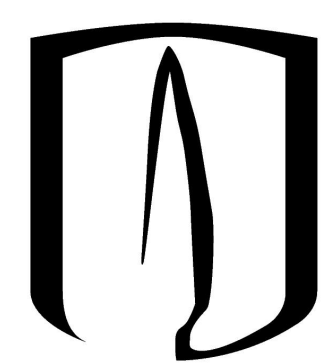

Departamento de Matemáticas

Facultad de Ciencias

Universidad de los Andes

Colombia

2014 


\section{Acknowledgements}

I would like to thank my advisor Mauricio Velasco for the time and effort he dedicated to this project and also for the astounding support, encouragement and patience. I also thank Greg Blekherman and Tristram Bogart for reading the document. 


\section{Abstract}

In this work, we study test sets for nonnegativity of real multivariate polynomials that are invariant under the action of a finite reflection group. In the first chapter we introduce some basic terminology, and some basics of finite reflection groups and their invariant theory. In the second chapter we present our results: we show that the hyperplane arrangement corresponding to a finite reflection group is a test set for polynomials invariant under it and which satisfy either a sparsity condition or have sufficiently small degree. We then conjecture how to further exploit the sparsity and degree to give lower dimensional test sets consisting of faces of the hyperplane arrangement. Finally, we study another method, consisting of restricting the attention to a certain subspace of invariant forms, to produce lower dimensional (still in the hyperplane arrangement) test sets. 


\section{Contents}

1 Preliminaries 4

1.1 Motivation . . . . . . . . . . . . . . . . . 4

1.2 Real Forms and Test Sets . . . . . . . . . . . . . . . . 6

1.3 Symmetric Polynomials . . . . . . . . . . . . . . . . . . 6

1.4 Finite Reflection Groups $\ldots \ldots \ldots$. . . . . . . . . . . . . 9

1.4 .1 Root Systems and Finite Reflection Groups $\ldots \ldots \ldots$

1.4 .2 Fundamental Domains and Isotropy Groups $\ldots \ldots \ldots \ldots$

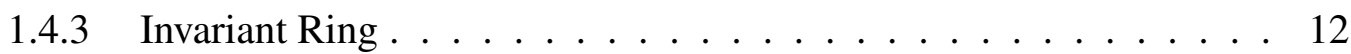

2 The Hyperplane Arrangement, Jacobians, Sparsity, and a Degree Principle 15

2.1 Factorization of the Jacobian and Test Sets . . . . . . . . . . . . 16

2.2 Sparsity and Minors of the Jacobian Matrix $\ldots \ldots \ldots \ldots$

2.3 A Half-Degree Principle? . . . . . . . . . . . . . . . . . . 20

2.4 More Jacobians, Harris Bases, and reducing the dimension of test sets . . . 22

2.4 .1 Restriction to subspaces . . . . . . . . . . . . . . 25

2.5 Future Directions . . . . . . . . . . . . . . . . . . . 30 


\section{Chapter 1}

\section{Preliminaries}

\subsection{Motivation}

The problem of deciding whether a polynomial is nonnegative has been one of the guiding questions of real algebraic geometry since the second half on XIX century. The intrinsic interest of this question has been complemented by a recent surge of possible applications, ranging from polynomial optimization [3] and control [18] to the general theory of approximation algorithms [30], among others. For all these applications, finding simple and efficient certificates for guaranteeing the nonnegativity of a given polynomial has become of paramount importance. Let's take a closer look. The optimization problem

$$
\min _{x \in S} f(x)
$$

where $f(x):=f\left(x_{1}, \ldots, x_{n}\right)$ is an $n$-variate real polynomial and $S \subset \mathbb{R}^{n}$, appears all around applied mathematics [18] and it's a hard computational problem: even when $f$ has degree 4 it can be NP-hard [7]. This problem is equivalent (i.e. has the same optimal value) to the following one

$$
\begin{aligned}
f^{*}:=\max _{\gamma \in \mathbb{R}} & \gamma \\
\text { s.t } & f(x)-\gamma \quad \text { is nonnegative on } S .
\end{aligned}
$$

Thus checking nonnegativity is fundamental for solving this problem. The most obvious way to decide whether a polynomial is nonnegative is by checking if it admits a representation as a sum of squares. However, not all nonnegative polynomials are sums of squares, David Hilbert proved in 1888 that the sets of nonnegative polynomials and sums of squares are the same in exactly three cases: univariate, quadratic and bivariate quartics [11]. Thus, in general, the problem (1.2), in the case $S=\mathbb{R}^{n}$, can be strictly relaxed to 


$$
\begin{aligned}
f_{\text {sos }}:=\max _{\gamma \in \mathbb{R}} & \\
\text { s.t } & f(x)-\gamma \text { is a sum of squares. }
\end{aligned}
$$

Despite the result of Hilbert, and the fact that in some cases $f_{\text {sos }}$ does not exist, this relaxation works rather well in practice [22]. In fact, this relaxed problem becomes a semidefinite optimization problem [21] and therefore can be solved efficiently. But still for large degree and number of variables it is computationally hard to solve with available methods [?]. Although, when $f$ is a polynomial that is invariant under the action of a finite group, the problem (1.3) can be simplified using representation theory of groups [9]. Another relaxation giving interesting lower bounds for $f^{*}$ which can be computed a lot faster than $f_{\text {sos }}$, and for larger instances, has arised using geometric programming [8]. In some cases, those bounds are at least as good as $f_{\text {sos }}$ [14]. But, to our knowledge, little is known about the gaps between the problem (1.2) and these relaxations. For instance, for fixed even degree and increasing number of variables, there are significantly more nonnegative polynomials than sums of squares [2], which seems to imply that the gap $f^{*}-f_{\text {sos }}$ can grow indefinitively as the number of variables increases. Perhaps, we should parallely consider alternatives for solving problem (1.2) that make no use of any relaxation. A convincing alternative is to find, independently of $f$, a significantly smaller subset of $S$ (and significantly smaller than a dense subset of $S$ ) where nonnegativity of $f$ is equivalent to nonnegativity of $f$ on $S$. To our knowledge, these "witness" subsets do not exist for general polynomials. But they do exist for some special families of symmetric polynomials:

Theorem 1.1 (Choi-Lam-Reznick [4]). An n-ary even symmetric sextic is nonnegative if and only if it is nonnegative on the $n$ points $(1,0 \ldots, 0),(1,1,0, \ldots, 0), \ldots,(1,1 \ldots, 1)$.

Theorem 1.2 (Harris [10]). An n-ary even symmetric octic is nonnegative if and only if it is nonnegative on the set of points whose components belong to a set $\{0,1, r\}$ for $r \in \mathbb{R}_{+}$.

Theorem 1.3 (Timofte's half-degree principle [32]). An n-variate symmetric polynomial of degree $2 d$ is nonnegative if and only if it is nonnegative on every point with at most $\max \{2, d\}$ distinct components.

In this document we study the problem of deciding nonnegativity of polynomials that are invariant under the action of a finite reflection group. We will not consider the sum of squares problem or any other relaxation of an optimization problem (although it would be an interesting project), we focus on witness subsets of $\mathbb{R}^{n}$ where nonnegativity of such polynomials implies their global nonnegativity. If the witness set is sufficiently simple, then checking nonnegativity becomes a simpler problem. In some cases, as we have seen above, those sets are dramatically smaller than the ambient space, for example a finite union of proper subspaces. 


\subsection{Real Forms and Test Sets}

In general we will use the letter $x$ to denote an $n$-tuple of variables $\left(x_{1}, \ldots, x_{n}\right)$, and the letters $y$ and $z$ to denote points $\left(y_{1}, \ldots, y_{n}\right),\left(z_{1}, \ldots, z_{n}\right)$ in $\mathbb{R}^{n}$. We will denote by $R$ the ring $\mathbb{R}\left[x_{1}, \ldots, x_{n}\right]$ of $n$-variate real polynomials. A nonconstant polynomial $f(x)$ is called a form if it is an homogeneous polynomial, i.e., all of its monomials have the same degree, or equivalently $f(r x)=r^{\operatorname{deg} f} f(x)$ for all real $r$. In other words, a form of degree $d \geq 1$ is an element of the graded component $R_{d}:=\mathbb{R}\left[x_{1}, \ldots, x_{n}\right]_{d}$ with respect to the usual grading of $R$ given by $\operatorname{deg} x_{i}=1$ for all $i=1, \ldots, n$, i.e., $R_{d}$ is the set of all homogeneous polynomials of degree $d$ inside $R$; and we have the decomposition $R=\bigoplus_{d=0}^{\infty} R_{d}$, where $R_{0}:=\mathbb{R}$. We will be working with forms because it will make our exposition clearer. We can switch between homogeneous and non-homogeneous polynomials by homogenization and dehomogenization; for example, the homogenization of $f(x)$ is the homogeneous polynomial $\bar{f}(x, t):=t^{\operatorname{deg} f} f\left(\frac{x}{t}\right)$ where a new variable $t$ is introduced; a homogenous polynomial can be dehomogenized in several ways just by evaluating one of the variables that appear (i.e., appears with nonzero coefficient) in the polynomial. A nonnegative polynomial is a polynomial with real coefficients that is greater or equal than zero when evaluated at every point of its domain. For example, an $n$-variate polynomial $f$ is nonnegative if $f(x) \geq 0$ for all $x \in \mathbb{R}^{n}$. For brevity, we will sometimes say that a polynomial is $p s d$ (positive semidefinite) which means exactly the same as "nonnegative". We do not lose anything in working with forms because a polynomial is psd if and only if its homogeneization is psd.

Definition 1.4. Let $A$ be a set of $n$-variate forms. We say that $\Omega \subset \mathbb{R}^{n}$ is a test set for $A$ if $f \in A$ if and only if $f(x) \geq 0$ for all $x \in \Omega$.

For instance, by continuity and homogeneity, any dense subset of $S^{n-1}$ is a test set for the set of nonnegative $n$-ary forms of degree $2 d$ (for any $d \geq 1$ ). But checking nonnegativity at it is as complex as checking nonnegativity at some dense subset of $\mathbb{R}^{n}$. We are interested in test sets which reduce the complexity of checking nonnegativity or at least are, somehow, substantially smaller than the ambient space. At least for a large family of symmetric polynomials there exist this kind of test sets [4, 10, 13, 32].

\subsection{Symmetric Polynomials}

A polynomial $p$ is called symmetric if it remains unchanged after permuting its variables. That is, $p\left(x_{1}, \ldots, x_{n}\right)=p\left(x_{\sigma(1)}, \ldots, x_{\sigma(n)}\right)$ for all permutations $\sigma$ belonging to the symmetric group on $n$ letters $S_{n}$. Here we consider the more general case of polynomials which have "symmetries", i.e, polynomials which are invariant under some nontrivial special action of a group. Consider for example a subgroup $G$ of the group $\mathrm{GL}(n, \mathbb{F})$ of linear automorphisms of $\mathbb{F}^{n}$, for some field $\mathbb{F}$. Then $G$ acts naturally on $\mathbb{F}^{n}$ and we say a polynomial $f \in \mathbb{F}\left[x_{1}, \ldots, x_{n}\right]$ is $G$-invariant if $f(g x)=f(x)$ for all $g \in G$. For example, we 
can see the $n$-variate symmetric polynomials as the polynomials invariant under the action of the group of permutation matrices inside $\operatorname{GL}(n, \mathbb{F})$. Since we are concerned with nonnegativity we will consider $G$-invariant real polynomials with respect to a subgroup $G$ of $\operatorname{GL}(n, \mathbb{R})$; also we consider the case of $G$ finite [explain why]. We will be concerned with the set of $G$-invariant polynomials inside $R$ which we will denote by $R^{G}$. This set is clearly a subring of $R$ since it is closed under addition and multiplication, and it is thus called the invariant subring of $R$ with respect to $G$, or for brevity, the invariant subring of $G$.

Theorem 1.5 (Hilbert-Noether, Chapter 16, [15]). Let $V$ be an n-dimensional vector space over a field $\mathbb{F}$. Let $G \subset \mathrm{GL}(V)$ be a finite group. Then the extension $\mathbb{F}\left[x_{1}, \ldots, x_{n}\right]^{G} \subset$ $\mathbb{F}\left[x_{1}, \ldots, x_{n}\right]$ is integral and $\mathbb{F}\left[x_{1}, \ldots, x_{n}\right]^{G}$ is a finitely generated $\mathbb{F}$-algebra.

Since $\mathbb{F}[x]$ is a finitely generated $\mathbb{F}$-algebra, both statements of the previous theorem imply that the extension $\mathbb{F}[x]^{G} \subset \mathbb{F}[x]$ is in fact finite. By Noether's normalization Lemma we can find a polynomial algebra $\mathbb{F}\left[y_{1}, \ldots, y_{m}\right]$ inside $\mathbb{F}[x]^{G}$ such that the extension $\mathbb{F}\left[y_{1}, \ldots, y_{m}\right] \subset \mathbb{F}[x]^{G}$ is finite. By the previous theorem, the integrality of the extension $\mathbb{F}[x]^{G} \subset \mathbb{F}[x]$ implies that both rings have the same Krull dimension and therefore $m=n$. Also, if the group $G$ is nonmodular, i.e., the characteristic of $\mathbb{F}$ does not divide $|G|$, then the extension $\mathbb{F}\left[y_{1}, \ldots, y_{n}\right] \subset \mathbb{F}[x]^{G}$ is finite and free, i.e., the ring $\mathbb{F}[x]^{G}$ is Cohen-Macaulay:

Theorem 1.6 (Hochster-Eagon, Chapter 16, [15]). Let $G \subset \mathrm{GL}(V)$ be a finite nonmodular group. Then $\mathbb{F}[x]^{G}$ is Cohen-Macaulay.

Let $R_{d}^{G}$ denote $d$-th graded component of $R^{G}$, i.e., the set of all $G$-invariant forms of degree $d$ inside $R$, then $R^{G}=\bigoplus_{d=0}^{\infty} R_{d}^{G}$ where $R_{0}^{G}=\mathbb{R}$. The $R_{d}^{G}$ 's will be central in this document (when $G$ is a $\mathrm{r}$ group), and our goal is to produce simple test sets for each of them. The graded components of a graded algebra can be seen as vector spaces over the field of definition, in particular, $R_{d}$ and $R_{d}^{G}$ are finite-dimensional (generated by the corresponding monomials of degree $d$ ) vector spaces over $\mathbb{R}$. Their dimension can be encoded algebraically: the Hilbert series of a graded algebra $A$ is the generating function $H(A, t)=\sum_{d=0}^{\infty} \operatorname{dim}\left(A_{d}\right) t^{d}$ where $\operatorname{dim} A_{d}$ is the dimension (as a vector space) of the $d$-th graded component over the defining field of $A$. For example, we can compute the Hilbert series of $R$ by calculating the dimension of each $R_{d}$ by counting the number of nonnegative integer tuples $\left(a_{1}, \ldots, a_{n}\right)$ that satisfy $a_{1}+\ldots+a_{n}=d$. This is clearly the coefficient of $t^{d}$ in the expression

$$
\left(1+t+t^{2}+t^{3}+\ldots\right)^{n}
$$

therefore $H(R, t)=\frac{1}{(1-t)^{n}}$.

Example 1.7. Let $R^{G}$ be a polynomial ring (which, as we will see, is very rare) and let $f_{1}, \ldots, f_{m}$ be algebraically independent generators for $R^{G}$ of degrees $e_{1}, \ldots, e_{m}$ respectively. The Hilbert series of $R^{G}$ is then given by

$$
H\left(R^{G}, t\right)=\frac{1}{\left(1-t^{e_{1}}\right) \ldots\left(1-t^{e_{m}}\right)}
$$


For $R^{G}$ we can compute its Hilbert series in terms of the matrices in $G$ with the following formula proved by Theodor Molien in 1897:

Theorem 1.8 ( [31], Theorem 2.2.1). The Hilbert series of the invariant ring $R^{G}$ equals

$$
H\left(R^{G}, t\right)=\frac{1}{|G|} \sum_{g \in G} \frac{1}{\operatorname{det}(i d-t g)}
$$

Molien's formula can be used to compute generators for $R^{G}$, however, the set of generators for $R^{G}$ can be very complex [31, Proposition 2.1.5]. We won't show those complicated examples but instead some at the other extreme.

Example 1.9. Let $\Lambda_{n, d}$ be the set of partitions of $d$ with at most $n$ parts, i.e., the set of $n$-tuples of nonnegative integers $\lambda=\left(\lambda_{1}, \ldots, \lambda_{n}\right)$ where $\lambda_{1} \geq \lambda_{2} \geq \ldots \geq \lambda_{n}$ and whose sum is $d$. We have distinct explicit bases for $R_{d}^{S_{n}}$ indexed by the partitions $\lambda$ in $\Lambda_{n, d}$, some of them are:

1. The monomial symmetric functions $m_{\lambda}$

$$
m_{\lambda}(x):=\sum_{\alpha} x_{1}^{\alpha_{1}} \ldots x_{n}^{\alpha_{n}}
$$

where $\alpha \in \mathbb{N}^{n}$ runs over all distinct permutations of $\lambda_{1}, \ldots, \lambda_{n}$. For example, take the partition $(3,1)$, then $m_{(3,1)}\left(x_{1}, x_{2}, x_{3}\right)=x_{1}^{3} x_{2}+x_{1} x_{2}^{3}+x_{1}^{3} x_{3}+x_{1} x_{3}^{3}+x_{2}^{3} x_{3}+x_{2} x_{3}^{3}$.

2. The power sum symmetric functions $p_{\lambda}$

$$
p_{\lambda}:=p_{\lambda_{1}} \cdots p_{\lambda_{k}}
$$

where $p_{r}$ is the $r$-th power sum defined by $p_{r}(x)=x_{1}^{r}+\ldots+x_{n}^{r}$ for $r$ a natural number.

3. The Schur functions $s_{\lambda}$

$$
s_{\lambda}(x):=\frac{D_{\lambda}}{\Delta_{n}}
$$

where

$$
D_{\lambda}:=\operatorname{det}\left[\begin{array}{cccc}
x_{1}^{\lambda_{1}+n-1} & x_{1}^{\lambda_{2}+n-2} & \ldots & x_{1}^{\lambda_{n}} \\
\vdots & \vdots & \ddots & \vdots \\
x_{n}^{\lambda_{1}+n-1} & x_{n}^{\lambda_{2}+n-2} & \ldots & x_{n}^{\lambda_{n}}
\end{array}\right]
$$


and $\Delta_{n}:=\prod_{1 \leq i<j \leq n}\left(x_{i}-x_{j}\right)$ is the Vandermonde determinant

$$
\Delta_{n}:=\operatorname{det}\left[\begin{array}{cccc}
x_{1}^{n-1} & x_{1}^{n-2} & \ldots & 1 \\
\vdots & \vdots & \ddots & \vdots \\
x_{n}^{n-1} & x_{n}^{n-2} & \ldots & 1
\end{array}\right]
$$

It is well known that Schur functions are symmetric polynomials and are the characters of the complex irreducible polynomial representations of $\operatorname{GL}(n, \mathbb{C})$. Moreover, they have a nice combinatorial interpretation:

Proposition 1.10 ( [28], Section 7.10).

$$
s_{\lambda}=\sum_{\mu} K_{\lambda \mu} m_{\mu}
$$

where the $K_{\lambda \mu}$ are the Kostka numbers, i.e, the number of semistandard Young tableaux of shape $\lambda$ and content $\mu$.

Now we proceed to our case of interest, where the ring $R^{G}$ is sufficiently simple.

\subsection{Finite Reflection Groups}

Let $V$ be a vector space over a field $\mathbb{F}$. A pseudo-reflection group is a subgroup $G \subset \operatorname{GL}(V)$ which is generated by pseudo-reflections, i.e. linear transformations whose pointwise fixed set is precisely an hyperplane (or whose eigenspace corresponding to the eigenvalue 1 is an hyperplane). A reflection group is a pseudo-reflection group generated by reflections, i.e. pseudo-reflections whose other eigenvalue is -1 . Since we are interested in finite groups that act on the ring $\mathbb{R}\left[x_{1}, \ldots, x_{n}\right]$, we set $\mathbb{F}=\mathbb{R}$ and consider finite pseudo-reflection groups $G \subset \mathrm{GL}(V)$. These are in fact finite reflection groups: the characteristic polynomial of a pseudo-reflection $g \in G$ is real and it has at most one imaginary root, thus it cannot have any; since $G$ is finite, the eigenvalues of a pseudo-reflection are roots of unity, thus the other eigenvalue is -1 . Note that reflections are orthogonal transformations, hence, reflection groups are subgroups of the orthogonal linear group $O(V)$. A reflection group $W$ is reducible if it can be decomposed as the direct product $W=W_{1} \times W_{2}$ where $W_{1}$ and $W_{2}$ are nontrivial reflection subgroups of $W$, otherwise $W$ is irreducible. If the only fixed point of $V$ under the action of $W \subset \mathrm{GL}(V)$ is the origin, we call $W$ essential. In the following section, we will work with (real) finite reflection groups which are essential.

\subsubsection{Root Systems and Finite Reflection Groups}

Let $V$ be a finite-dimensional Euclidean vector space, with the standard Euclidean inner product denoted by $(\cdot, \cdot)$. A root system in $V$ is a finite set $\Phi$ of non-zero vectors (called roots) that satisfy the following conditions: 
(R1) The only scalar multiples of a root $\alpha \in \Phi$ that belong to $\Phi$ are $\alpha$ and $-\alpha$.

(R2) For every root $\alpha \in \Phi$, the set $\Phi$ is invariant under reflection through the hyperplane orthogonal to $\alpha$.

It is to be understood that the hyperplanes above pass through the origin. For a root $\alpha$ we call the corresponding orthogonal hyperplane $H_{\alpha}$ and the corresponding reflection through this hyperplane $s_{\alpha}$. The reflection group $W$ generated by them is called the reflection group of $\Phi$. The number of reflections in $W$ is precisely the number of hyperplanes $H_{\alpha}[12$, Proposition 1.14], and therefore half the number of roots. Since to each reflection corresponds and hyperplane of reflection, we will say that the $H_{\alpha}$ are the hyperplanes of $W$. One of the main characters of this work is the union of those hyperplanes:

$$
\mathcal{H}_{W}:=\bigcup_{\alpha \in \Phi} H_{\alpha}
$$

which is an hyperplane arrangement [5], and we will call it sometimes the arrangement. Observe that a reflection group arising from a root system is finite since it acts faithfully on the finite set $\Phi$. We say that the root system $\Phi$ has $\operatorname{rank} n$ if $\operatorname{dim} \operatorname{span} \Phi=n$. If a root system $\Phi$ satisfies the additional condition:

(R3) The roots in $\Phi$ span $V$

then it is called essential. Note that only when $\Phi$ is essential the intersection of the $H_{\alpha}$ is just the origin, and therefore $W$ acts in $V$ with no nonzero fixed points, i.e., $W$ is essential.

Example 1.11. Dihedral groups $I_{2}(m)$. Drawings?

Irreducible reflection groups are completely classified: there are four one-parameter families $A_{n}, B C_{n}, D_{n}, I_{2}(m)$ and seven exceptional groups $E_{6}, E_{7}, E_{8}, F_{4}, G_{2}, H_{3}$ and $H_{4}[12$. Chapter 2]. If additionally, a root system satisfies the condition

(R4) $2 \frac{(\alpha, \beta)}{(\beta, \beta)}$ is an integer for every pair of roots $\alpha, \beta \in \Phi$.

then it is called a crystallographic root system, and the corresponding reflection group generated is called a Weyl group. Crystallographic root systems appear in the study of semisimple Lie algebras, they are completely classified and therefore Weyl groups too: there are three one-parameter families $A_{n}, B C_{n}, D_{n}$ and five exceptional groups $E_{6}, E_{7}, E_{8}, F_{4}$ and $G_{2}$. Despite our main interest is on reflection groups we will use Weyl groups as a source of examples. We will say that $W$ is of rank $n$ if $W$ is a reflection group such that $W \subset \mathrm{GL}(n, \mathbb{R})$ or if it arises from a root system (or crystallographic root system) of rank $n$. 


\subsubsection{Fundamental Domains and Isotropy Groups}

We can define a total order $<$ on $V$ and define $\Phi_{<}^{+}$to be the set of roots $\alpha \in \Phi$ such that $0<\alpha$ in the order $<$. We call $\Phi_{<}^{+}$a positive system and simply write $\Phi^{+}$when we refer to a positive system without regarding the order. A total order can be given to $V$ : choose an arbitrary ordered basis $v_{1}, \ldots, v_{n}$ of $V$ and adopt the lexicographic order, where $\sum a_{i} v_{i}<\sum b_{i} v_{i}$ means that $a_{k}<b_{k}$ if $k$ is the least index $i$ for which $a_{i} \neq b_{i}$. Since roots come in pairs, $\Phi$ is the disjoint union of $\Phi^{+}$and $-\Phi^{+}$, this implies that the set of hyperplanes $\left\{H_{\alpha}: \alpha \in \Phi^{+}\right\}$is the same regardless of the order.

Definition 1.12. A subset $\Delta$ of $\Phi$ is called a simple system if it is a vector space basis for the $\mathbb{R}$-span of $\Phi$ in $V$ and if each $\alpha \in \Phi$ is a linear combination of $\Delta$ with coefficients all of the same sign (all nonnegative or all nonpositive).

It is not evident that simple systems exist, but they always do [12, Theorem 1.3]. In fact, we can count exactly how many there are: since the action of $W$ on simple systems is simply transitive [12, Theorem 1.8], the number of simple systems equals $|W|$.

Fix a simple system $\Delta$ inside $\Phi$ and let $S:=\left\{s_{\alpha}: \alpha \in \Delta\right\}$ be the set of reflections corresponding to roots in $\Delta$, which we call simple reflections. For a subset $I \subset S$, denote by $\Delta_{I}$ the set of roots in $\Delta$ corresponding to reflections in $I$. Let $\Phi_{I}$ the intersection of $\Phi$ with the $\mathbb{R}$-span $V_{I}$ of $\Delta_{I}$ in $V$. It follows that $\Phi_{I}$ is a root system in $V_{I}$ and its reflection group $W_{I}$ is generated by the reflections in $I$ [12, Proposition 1.10]. Note that $W_{I}$ is essential if seen as acting in $V_{I}$, not in $V$. The subgroups $W_{I}$ of $W$, obtained by varying $I$ and $\Delta$, are called parabolic subgroups. We think they will be very important in our work because they facilitate inductive arguments.

Associated to $\Delta$ we have the fundamental domain $D:=\{v \in V:(v, \alpha) \geq 0$ for all $\alpha \in$ $\Delta\}$ which is the closure of the intersection $C:=\bigcap_{\alpha \in \Delta} H_{\alpha}^{+}$of the open half spaces $H_{\alpha}^{+}:=\{v \in V:(v, \alpha)>0\}$ associated to the hyperplanes $H_{\alpha}$ for each root $\alpha \in \Delta$. $D$ is a closed convex pointed polyhedral cone with non-empty interior, i.e, a polyhedral proper cone. Note the number of walls or facets, i.e. faces of maximal dimension, of $D$ is $\operatorname{dim} V$. The reason for the name of $D$ is the following: for each $v \in V \backslash\{0\}$ there is a unique element $u \in D$ such that $u=w v$ for some $w \in W$ [12, Lemma 1.12], i.e., the orbit $W v:=\{w v: w \in W\}$ of $v$, under the action of $W$, intersects exactly once with $D$. The set $\{w C: w \in W\}$ partitions $\mathbb{R}^{n} \backslash \mathcal{H}_{W}$ in open connected components, which we call chambers. To each chamber $C$ we can associate a simple system by taking the roots that are orthogonal to its walls (the walls of its closure) and positively oriented (i.e. such that $C$ is in the same side of the root with respect to the wall). The simply transitive action of $W$ on simple systems induces a simply transitive action of $W$ on chambers, therefore, the number of chambers equals the order of $W$. To summarize, we have: 
Proposition 1.13. The set $\mathbb{R}^{n} \backslash \mathcal{H}_{W}$ consists of $|W|$ open connected components in which $W$ acts transitively, each of which its closure is a polyhedral proper cone with exactly $n$ facets.

The $k$-dimensional faces of the fundamental domain $D$ are the intersection of exactly $n-k$ facets of $D$. For instance, the $k$-dimensional faces of $D$ are precisely the

$$
C_{I}:=\left\{u \in D:(u, \alpha)=0 \text { for all } \alpha \in \Delta_{I},(u, \alpha)>0 \text { for all } \alpha \in \Delta \backslash \Delta_{I}\right\}
$$

where $I$ varies over all subsets of $S$ of size $n-k$.

Definition 1.14. The isotropy group $W_{A}$ of a subset $A \subset V$ is the set of elements of $W$ which fix $A$ pointwise, i.e., $W_{A}=\{w \in W: w a=a$ for all $a \in A\}$.

Clearly, an isotropy group is a subgroup of $W$. It is easy to see that the isotropy group of the face $C_{I}$ is precisely the parabolic subgroup $W_{I}$ :

Proposition 1.15 ( [12], Proposition 1.15). For each $I \subset S$, the isotropy group of the face $C_{I}$ is precisely $W_{I}$.

Proof. From the definition of $C_{I}$, it is clear that $W_{I}$ fixes it pointwise. If for $w \in W$ it happens that $w C_{I}=C_{I}$ then, by [12, Theorem 1.12], $w$ belongs to $W_{I}$.

\subsubsection{Invariant Ring}

Clearly, if a polynomial is invariant under the action of a set of generators of $G$ it will be $G$-invariant. In particular, if a root system $\Phi$ gives rise to a reflection group $W$, then the set of reflections $\left\{s_{\alpha}: \alpha \in \Phi\right\}$ generates $W$, therefore, if a polynomial $f$ is invariant under every $s_{\alpha}$ it will be $W$-invariant.

Example 1.16. The square of the norm $\|x\|^{2}=x_{1}^{2}+\ldots+x_{n}^{2}$ is a polynomial invariant under any reflection through a hyperplane that passes through the origin since norm is preserved by an orthogonal transformation, therefore it is $W$-invariant for any reflection group $W$.

The following theorem is one of the reasons why we consider reflection groups, it says that the invariant ring is particularly simple precisely for matrix groups generated by pseudo-reflections.

Theorem 1.17 ( [31], [20], Shephard-Todd-Chevalley). The invariant ring $R^{W}$ is generated as a $\mathbb{R}$-algebra by $n$ algebraically independent homogeneous invariants of positive degree (together with 1) if and only if $W$ is a pseudo-reflection group. Moreover, $R$ is a free $R^{W}$-module of rank $|W|$. 
From now on we suppose that $W$ is a finite (real) reflection group of rank $n$.

Remark. It is important to notice that being a reflection group is not a property of the abstract group underlying $W$ but it depends on the specific $n$-dimensional representation. For instance, the dihedral group $D_{6}$ has faithful 2-dimensional representation which is a reflection group, but it also has a faithful 3-dimensional representation which is not a reflection group [31, Example 2.2.6]. With that in mind is fair to ask if every finite group admits a faithful representation that is a reflection group, but that is not the case since the center of an irreducible reflection group is cyclic.

Definition 1.18. A set of basic invariants for $W$ is a set of algebraically independent homogeneous generators for the algebra $R^{W}$.

As we have seen, there are different choices for a set of basic invariants for $W$ (Example 1.9], but the multiset of their degrees is unique [12, Proposition 3.7], so we will call them the degrees of $W$. Moreover, we have the following formulas, for which we present the proof given at [12, Theorem 3.9]:

Theorem 1.19. Let $d_{1}, \ldots, d_{n}$ be the degrees of $W$, and $N$ the number of reflections in $W$. Then $|W|=d_{1} \ldots d_{n}$ and $N=\left(d_{1}-1\right)+\ldots+\left(d_{n}-1\right)$.

Proof. By 1.5 and Example 1.7 we have

$$
\frac{1}{|W|} \sum_{w \in W} \frac{1}{\operatorname{det}(i d-t w)}=\frac{1}{\left(1-t^{d_{1}}\right) \ldots\left(1-t^{d_{n}}\right)}
$$

Multiplying both sides by $(1-t)^{n}$ and observing that the elements in $W$ with exactly $n-1$ eigenvalues equal to 1 are precisely the reflections, we obtain

$$
\frac{1}{|W|}\left(1+N \frac{1-t}{1+t}+(1-t)^{2} g(t)\right)=\prod_{i=1}^{n} \frac{1}{1+t+\ldots+t^{d_{i}-1}}
$$

where $g(t)$ is a rational function with denominator not divisible by $1-t$. Evaluating this at $t=1$, we get

$$
\frac{1}{|W|}=\frac{1}{d_{1} \ldots d_{n}}
$$

or $|W|=d_{1} \ldots d_{n}$. Differentiating 1.9 , we get

$$
-\frac{2 N}{|W|} \frac{1}{(1+t)^{2}}+h(t)=\left(\prod_{i=1}^{n} \frac{1}{1+t+\ldots+t^{d_{i}-1}}\right)\left(\sum_{i=1}^{n}-\frac{1+2 t+\ldots+\left(d_{i}-1\right) t^{d_{i}-2}}{1+t+\ldots+t^{d_{i}-1}}\right)
$$


where $h(t)$ is a rational function with numerator divisible by $1-t$. Evaluating this at $t=1$, we get

$$
-\frac{N}{2|W|}=-\frac{1}{2} \frac{1}{d_{1} \ldots d_{n}} \sum_{i=1}^{n}\left(d_{i}-1\right)
$$

and using that $|W|=d_{1} \ldots d_{n}$ we obtain $N=\left(d_{1}-1\right)+\ldots+\left(d_{n}-1\right)$.

Remark. If $W$ is essential then it cannot have an invariant polynomial of degree 1 because that will imply that $W$ fixes a hyperplane. So if $W$ is essential with degrees $d_{1} \leq d_{2} \leq$ $\ldots \leq d_{n}$ we should have, by Example $1.16, d_{1}=2$.

In order for a set of homogeneous polynomials in $R^{W}$ of degrees $e_{1}, \ldots, e_{n}$ to be basic invariants for $W$ we only need to check two things: $\prod e_{i}=|W|$ and algebraic independence [12, Proposition 3.12]. For the latter, we have the following criterion:

Proposition 1.20. The polynomials $f_{1}, \ldots, f_{n} \in K\left[x_{1}, \ldots, x_{n}\right]$ are algebraically independent (over a field $K$ of characteristic 0 ) if and only if the Jacobian $\operatorname{det}\left(\nabla f_{1}, \ldots, \nabla f_{n}\right)$ is not identically zero.

In what follows, we will write $v_{1} \wedge \ldots \wedge v_{r}$ for the determinant $\operatorname{det}\left(v_{1}, \ldots, v_{r}\right)$ of the matrix whose columns are the vectors $v_{1}, \ldots, v_{r} \in \mathbb{R}^{r}$, in that order.

Example 1.21. The set of power sums $p_{2}, \ldots, p_{n+1}$ is a set of basic invariants for $A_{n}$. The even power sums $p_{2}, p_{4}, \ldots, p_{2 n}$ is a set of basic invariants for $B C_{n}$. By Proposition 1.20 , the Vandermonde determinant, seen as the jacobian of these sets of functions, proves both statements.

The following table contains the degrees of the basic invariants for each irreducible reflection group.

\begin{tabular}{|c|l|}
\hline Root System & Degrees $d_{i}$ \\
\hline$A_{n}$ & $2,3, \ldots, n+1$ \\
\hline$B C_{n}$ & $2,4,6, \ldots, 2 n$ \\
\hline$D_{n}$ & $2,4,6, \ldots, 2 n-2, n$ \\
\hline$E_{6}$ & $2,5,6,8,9,12$ \\
\hline$E_{7}$ & $2,6,8,10,12,14,18$ \\
\hline$E_{8}$ & $2,8,12,14,18,20,24,30$ \\
\hline$F_{4}$ & $2,6,8,12$ \\
\hline$G_{2}$ & 2,6 \\
\hline$H_{3}$ & $2,6,10$ \\
\hline$H_{4}$ & $2,12,20,30$ \\
\hline$I_{2}(m)$ & $2, m$ \\
\hline
\end{tabular}




\section{Chapter 2}

\section{The Hyperplane Arrangement, Jacobians, Sparsity, and a Degree Principle}

In this chapter we present our main results. We first introduce the Chevalley mapping and its Jacobian Matrix, from where the arrangement $\mathcal{H}_{W}(1.8)$ appears naturally as the vanishing locus of its determinant [Proposition 2.2]. This will allow us to show, using Lagrange multipliers, that each curve of a certain family intersects the arrangement [Lemma 2.5]. Surprisingly, for each $W$-invariant form we can consider a particular curve in the family above, such that among its points of intersection with the arrangement lies a minimizer of the form. This holds under a weak sparsity condition or a strong (but sharp) restriction on the degree of the form. As a result we conclude that $\mathcal{H}_{W}$ is a test set for a large family of $W$-invariant forms, and additionally show that it is not for general $W$-invariant forms of large degree [Theorems 2.6 and 2.13]. After that we show how to exploit stronger sparsity conditions on a $W$-invariant form, making use of the vanishing locus of the minors of the Jacobian matrix of the Chevalley map, to get even simpler test sets consisting of lower dimensional faces of the arrangement, and propose some conjectures. In the second last section we introduce Harris bases and use them to get simpler test sets in some cases, showing examples for $F_{4}$. Finally we propose some questions that we think could lead to new ways for attacking the decision problem of nonnegativity for $W$-invariant polynomials.

From now on, we will always assume the following:

1. $\Phi$ is a root system in $V$ with associated reflection group $W$ or vice versa, both of rank $n$.

2. $\eta_{1}, \ldots, \eta_{n}$ are a complete set of basic invariants for $W$ of degrees $d_{1} \leq \cdots \leq d_{n}$. 


\subsection{Factorization of the Jacobian and Test Sets}

Definition 2.1. Associated to a set of basic invariants $\eta_{1}, \ldots, \eta_{n}$ for a reflection group $W$ we have the Chevalley map $\Psi: \mathbb{R}^{n} \rightarrow \mathbb{R}^{n}$ sending $x$ to $\left(\eta_{1}(x) \ldots, \eta_{n}(x)\right)$. Call $J_{W}$ the Jacobian matrix of this map, i.e., the matrix whose columns are $\nabla \eta_{1}, \ldots, \nabla \eta_{n}$ in that order, and denote by $\left|J_{W}\right|$ its determinant.

The following is a classical result [12, Proposition 3.13] that will turn to be very useful for characterizing test sets for $W$-invariant polynomials.

Proposition 2.2. For each root $\alpha \in \Phi$, define $l_{\alpha}$ to be a (nonzero) linear form that vanishes at the hyperplane $H_{\alpha}$. Then

$$
\left|J_{W}\right|=c \prod_{\alpha \in \Phi^{+}} l_{\alpha}
$$

for some nonzero constant $c$.

We make use of the following lemma and present both proofs in [12] for completeness.

Lemma 2.3 (Lemma 3.3, [12]). Let $l$ be a linear form in $\mathbb{R}\left[x_{1}, \ldots, x_{n}\right]$ and suppose the polynomial $f$ vanishes at all zeros of $l$. Then $l$ divides $f$.

Proof. Suppose (without loss of generality) that $x_{n}$ occurs with a nonzero coefficient in $l$ and perform the division algorithm with respect to $x_{n}$ to obtain

$$
f=l q+r
$$

where $q \in \mathbb{R}\left[x_{1}, \ldots, x_{n}\right]$ and $r \in \mathbb{R}\left[x_{1}, \ldots, x_{n}\right]$ has degree 0 in $x_{n}$. Evaluating $[2.2]$ at the hyperplane in which $l$ vanishes we conclude that $r$ also vanishes in that hyperplane, and even more: at all $\mathbb{R}^{n}$ since $x_{n}$ can take any value. Therefore, $r \equiv 0$ and $l$ divides $f$.

Proof of Proposition 2.2 Suppose $a=\left(a_{1}, \ldots, a_{n}\right) \in H_{\alpha}$ for some root $\alpha$. Hence, every open neighborhood of $a$ contains a pair of distinct points $b, c$ for which $s_{\alpha} b=c$. But then $\eta_{i}(c)=\eta_{i}\left(s_{\alpha} b\right)=s_{\alpha} \eta_{i}(b)=\eta_{i}(b)$, forcing $\Psi(c)=\Psi(b)$. Therefore, $\left|J_{W}\right|$ must vanish at $a$, otherwise this will contradict the Implicit Function Theorem. By Lemma 2.3, we conclude that $l_{\alpha}$ divides $\left|J_{W}\right|$. Since for $\alpha \in \Phi^{+}$the $l_{\alpha}$ are irreducible and not proportional, we conclude that their product divides $\left|J_{W}\right|$. By Theorem 1.19 , the degree of this product coincides with the degree of the Jacobian and the conclusion follows. By Proposition 1.20 since basic invariants are algebraically independent, the constant $c$ is nonzero.

Therefore $\nabla \eta_{1} \wedge \ldots \wedge \nabla \eta_{n} \doteq \prod_{\alpha \in \Phi^{+}} l_{\alpha}$, where $a \doteq b$ means $a=\lambda b$ up to a nonzero constant $\lambda$. So $\left|J_{W}\right|$, contrary to $J_{W}$, does not depend (modulo $\doteq$ ) on the choice of basic invariants for $W$. 
Definition 2.4. We say that a point $v \in V$ is $W$-general if the orbit $W v$ of $v$ under the action of $W$ has maximal length, that is, $|W v|=|W|$ [Proposition 1.13], otherwise we say that $v$ is $W$-special. Equivalently, $v \in V$ is $W$-special if and only if it belongs to $\mathcal{H}_{W}$; or, by Proposition 2.2, $v$ is $W$-special if and only if the matrix $J_{W}(v)$ is singular.

With the following lemma we will be able to find $W$-special minimizers for certain $W$-invariant forms, we make use of the Lagrange multipliers idea from [26]. First we state an important "weak" compactness condition that must be satisfied in order for subsequent results to be true.

Condition 1. The form $\|x\|^{2}$ is contained in the algebra generated by a given set of polynomials.

Lemma 2.5. Let $\eta_{1}, \ldots, \eta_{n}$ be basic invariants for a reflection group $W$ and $y \in \mathbb{R}^{n} a$ $W$-general point. Let $\mathcal{C}_{y}^{r} \subset \mathbb{R}^{n}$ be the curve defined by the $n-1$ equations

$$
\eta_{1}(x)=\eta_{1}(y), \ldots, \eta_{r}\left(\widehat{x)=\eta_{r}}(y), \ldots, \eta_{n}(x)=\eta_{n}(y)\right.
$$

where $\eta_{r}\left(\widehat{x)=\eta_{r}}(y)\right.$ means that this equation is missing. Suppose that Condition 1 holds for these $n-1$ invariants. Then, for every $y \in \mathbb{R}^{n} \backslash\{0\}, \mathcal{C}_{y}^{r}$ contains a nonzero $W$-special point.

Proof. The curve $\mathcal{C}_{y}^{r}$ is closed, and, because Condition 1 holds, it's contained in the sphere of radius $\|y\|^{2}$, therefore it's compact. Hence, the function $\eta_{r}$ has an extremum $\zeta \in \mathcal{C}_{y}^{r}$ (which is not zero since $y$ is not zero) which must satisfy, by Lagrange's theorem, the following condition

$$
\left(1-\lambda_{r}\right) \nabla \eta_{r}(\zeta)+\sum_{i=1}^{n} \lambda_{i} \nabla \eta_{i}(\zeta)=0
$$

for some real numbers $\lambda_{1}, \ldots, \lambda_{n}$. In other terms, the vector $\lambda:=\left[\lambda_{1}, \ldots, 1, \ldots, \lambda_{n}\right]$ (where the 1 is at the $r$-th position) belongs to the kernel of the matrix $J_{W}(\zeta)$. By Proposition 2.2. $\left|J_{W}\right|$ vanishes only at $\mathcal{H}_{W}$, but since $0 \neq \lambda \in \operatorname{ker} J_{W}(\zeta)$, this implies $\left|J_{W}\right|$ vanishes at $\zeta$, in other words, $\zeta \in \mathcal{H}_{W}$, i.e., $\zeta$ is a $W$-special point in $\mathcal{C}_{y}^{r}$.

We will call the above curve $\mathcal{C}_{y}$ when we do not specifically say which invariant is missing in its definition. As a corollary, we have the following theorem which says that $\mathcal{H}_{W}$ is a test set for weakly "sparse" forms, independent of the degree.

Theorem 2.6. Let $f$ be an $n$-ary $W$-invariant form of degree $2 d$ belonging to the algebra generated by no more than $n-1$ basic invariants for which Condition 1 holds. Then $f$ is nonnegative if and only if it is nonnegative on $\mathcal{H}_{W}$. 
Proof. Suppose $f$ is nonnegative on $\mathcal{H}_{W}$ but not nonnegative on $\mathbb{R}^{n}$. Let $\mu<0$ be the minimum of $f$ on the unit sphere $S^{n-1}$ and $y$ a $W$-general point in $S^{n-1}$ that realizes this minimum. Since Condition 1 holds, the corresponding curve $\mathcal{C}_{y}$ is contained in the unit sphere $S^{n-1}$. The form $g(x):=f(x)-\mu\|x\|^{2 d}$ is nonnegative and also depends on $n-1$ basic invariants, hence, there exists, by Lemma 2.5, a $W$-special point $z \in \mathcal{C}_{y}$ that satisfies $g(y)=g(z)=0$, giving $f(z)=\mu<0$, a contradiction.

Any $W$-invariant polynomial $f$ can be written as $f=g\left(\eta_{1}, \ldots, \eta_{n}\right)$ for a unique polynomial $g \in \mathbb{R}\left[X_{1}, \ldots, X_{n}\right]$. Let $J:=\left\{0<j_{1}<\ldots<j_{r}\right\} \subset \mathbb{N}$, we say $f$ is $J$-sparse if it can be written as $g\left(\eta_{j_{1}}, \ldots, \eta_{j_{r}}\right)$ for a polynomial $g \in \mathbb{R}\left[X_{1}, \ldots, X_{r}\right]$. In particular, any polynomial $f \in \mathbb{R}\left[x_{1}, \ldots, x_{n}\right]^{W}$ is at most $[n]$-sparse.

Definition 2.7. Let $f_{1}, \ldots, f_{m}$ be $n$-variate real polynomials. The basic semialgebraic set defined by $f_{1}, \ldots, f_{m}$ is the set $\left\{x \in \mathbb{R}^{n}: f_{1}(x) \geq 0, \ldots, f_{m}(x) \geq 0\right\}$ and we denote it by $S\left(f_{1}, \ldots, f_{m}\right)$.

Theorem 2.8. Let $f_{1}, \ldots, f_{m}$ be $W$-invariant $J$-sparse polynomials, with $J \subsetneq[n]$ such that either $|J| \leq n-2$ or Condition 1 holds for the forms in J. Then

$$
S\left(f_{1}, \ldots, f_{m}\right) \neq \emptyset \Longleftrightarrow S\left(f_{1}, \ldots, f_{m}\right) \cap \mathcal{H}_{W} \neq \emptyset
$$

Proof. The inclusion $C_{J, y}:=\left\{x \in \mathbb{R}^{n}: \eta_{j}(x)=\eta_{j}(y)\right.$ for all $\left.j \in J\right\} \subset S\left(f_{1}, \ldots, f_{m}\right)$ for $y \in S\left(f_{1}, \ldots, f_{m}\right)$, follows since $z \in C_{J, y}$ implies $\eta_{j}(z)=\eta_{j}(y)$ for each $j \in J$, and the hypothesis that the $f_{i}$ are $J$-sparse. If Condition 1 holds for the forms in $J$, the same argument in the proof of Lemma 2.5 implies that $C_{J, y} \cap \mathcal{H}_{W}$ is nonempty and therefore $S\left(f_{1}, \ldots, f_{m}\right) \cap \mathcal{H}_{W} \neq \emptyset$. If Condition 1 does not hold and $|J| \leq n-2$, then we can add $\|x\|^{2}$ to the definition of $C_{J, y}$ [Example 1.16].

\subsection{Sparsity and Minors of the Jacobian Matrix}

When $f$ is a $W$-invariant form of even degree belonging to the algebra generated by the first $k<n$ basic invariants $\eta_{1}, \ldots, \eta_{k}$, there is an even simpler test set: the $k$-dimensional faces of the arrangement $\mathcal{H}_{W}$, or equivalently, of a fundamental domain [Theorem 2.12]. The approach is the following:

By the same argument of the proof of Lemma 2.5, assuming Condition 1 holds for the first $k$ invariants, we have a point $\zeta$ that satisfies

$$
\nabla \eta_{k+1}(\zeta)+\sum_{i=1}^{k} \lambda_{i} \nabla \eta_{i}(\zeta)=0
$$

which implies that the matrix whose columns are $\nabla \eta_{1}(\zeta), \ldots, \nabla \eta_{k+1}(\zeta)$ has rank at most $k$, and then its maximal minors vanish. Therefore, following the proof of Theorem 2.6, the 
vanishing locus of the maximal minors of the matrix $J_{W, k+1}$ with columns $\nabla \eta_{1}, \ldots, \nabla \eta_{k+1}$ is a test set for this family of sparse forms. Thus it would be nice to have a better description of this locus.

The following result due to R. Steinberg takes us closer to the desired description.

Theorem 2.9 (Steinberg, [29]). Let $W$ be a finite pseudo-reflection group acting on a vector space $V$ of finite dimension. Let $p$ be any point of $V$. Then the following numbers are equal:

(a) the maximum number of linearly independent hyperplanes containing $p$,

(b) the maximum rank of $1-w$ for all $w$ in $W$ for which $w p=p$,

(c) the nullity of $J_{W}$ at $p$.

Note that (a) and (c) imply that $p$ is in the interior of a $k$-dimensional face of $\mathcal{H}_{W}$ if and only if the rank of $J_{W}(p)$ equals $k$.

Corollary 2.10. The vanishing locus of the ideal generated by the $(k+1) \times(k+1)$ minors of $J_{W}$ is precisely the set of $k$-dimensional faces of $\mathcal{H}_{W}$.

Proof. Let $p \in \mathbb{R}^{n}$. $p$ belongs to the vanishing locus of $(k+1) \times(k+1)$ minors $\Longleftrightarrow$ rank $J_{W}(p) \leq k \Longleftrightarrow p$ belongs to a $k$-dimensional face of $H_{W}$. Where the last equivalence is because of Theorem 2.9 .

It would be useful to know if the maximal minors of $J_{W, k}$ have a nice factorization and if it can be computed explicitly or in some coordinate-free fashion. Perhaps an argument along the following lines could be useful.

Proof. A point in a $k$-dimensional face of the fundamental domain $D$ belongs to $p \geq n-k$ hyperplanes $H_{\alpha_{1}}, \ldots, H_{\alpha_{p}}$ associated to the corresponding simple system $\Delta$. Points in $F:=H_{\alpha_{1}} \cap \cdots \cap H_{\alpha_{p}}$ have the same isotropy group $W_{F}$ (Proposition 1.15). As $W_{F}$ is a parabolic subgroup, and since $\Psi$ is $W_{F}$ invariant, we have $\Psi=Q \circ \Psi_{F}$ where $\Psi_{F}$ is a Chevalley mapping for $W_{F}$ and $Q$ is invertible. The rank of $\Psi$ on a $k$-dimensional face of $D$ is $k$ [1, Section 2], therefore, all $(k+1) \times(k+1)$ minors of $J_{W}$ vanish precisely on $F$.

To close this section we propose the following kind-of-stronger conjecture based on what happens for $A_{n}$ and $B C_{n}$ with the Jacobians of power sums (1.6). First a definition:

Conjecture 2.11. The vanishing locus of the $k \times k$ minors corresponding to the first $k$ columns of $J_{W}$ coincides with the vanishing locus of all $k \times k$ minors of $J_{W}$.

As a corollary of the results in this section, along with the conjecture, we have the desired result: 
Theorem 2.12 (Friedl, Riener, Sanyal [27]). Let $f \in R_{2 d}^{W}$ belong to the algebra generated by the first $k<n$ basic invariants $\eta_{1}, \ldots, \eta_{k}$, and suppose Condition 1 holds for them. Then $f$ is nonnegative if and only if it is nonnegative at the $k$-dimensional faces of $\mathcal{H}_{W}$.

\subsection{A Half-Degree Principle?}

We have exploited the algebraic sparsity to produce test sets and make conjectures. In this section we show that the hyperplane arrangement is also a test set for low degree $W$-invariant polynomials and show a sharpness result in that direction. We also conjecture what would be a generalization of Timofte's half-degree principle for finite reflection groups.

Theorem 2.13. Assume $\|x\|^{2}$ is an element of the algebra generated by the first $n-1$ invariants. If $f$ is a $W$-invariant form of degree $2 d<2 d_{n}$ then $f$ is nonnegative if and only if $f$ is nonnegative on $\mathcal{H}_{W}$.

incorrect. Note we can write

$$
f=g_{0}\left(\eta_{1}, \ldots, \eta_{n-1}\right)+g_{1}\left(\eta_{1}, \ldots, \eta_{n-1}\right) \eta_{n}
$$

For polynomials $g_{0}, g_{1}$ in $\mathbb{R}\left[Z_{1}, \ldots, Z_{n-1}\right]$. Let $y$ be a minimizer for $f$ on the unit sphere. Then the restriction $\phi$ of $f$ to the $\operatorname{set} \mathcal{C}_{y}^{n}$ is

$$
\phi=c_{0}+c_{n} \eta_{n}
$$

for constants $c_{0}, c_{n}$. Hence, a minimizer $x^{*}$ of $\phi$ in $\mathcal{C}_{y}^{n}$ must satisfy

$$
c_{n} \nabla \eta_{n}\left(x^{*}\right)=\sum_{k=1}^{n-1} c_{k} \nabla \eta_{k}\left(x^{*}\right)
$$

If not all $c$ 's are zero, then $x^{*} \in \mathcal{H}_{W}$ and therefore $\mathcal{H}_{W}$ is a test set. If all $c$ 's are zero this would imply that $f$ is constant on $\mathcal{C}_{y}^{n}$. Since $\mathcal{C}_{y}^{n}$ is nonempty, Theorem 2.8 implies $\mathcal{C}_{y}^{n} \cap \mathcal{H}_{W}$ is nonempty and therefore $\mathcal{H}_{W}$ is a test set.

Next, it is natural to ask whether the degree bound in Theorem 2.13 is sharp. We prove that this is the case when $W$ is a reflection group all of whose basic invariants have even degree (i.e. for those reflection groups containing multiplication by -1 ). More generally, we prove the following existence theorem for $W$-invariant forms for which $\mathcal{H}_{W}$ is not a witness set for nonnegativity.

Theorem 2.14. Let $\underline{o}$ and $\bar{o}$ be the smallest and largest odd degrees of basic invariants for $W$. Let $\underline{o}=\bar{o}=1$ if all invariants of $W$ have even degree. If $2 d \geq \max \left(2 d_{n}, 2(\underline{o}+\bar{o})\right)$ then there exists a $W$-invariant form $f$ of degree $2 d$ which is nonnegative on $\mathcal{H}_{W}$ and which is negative at some point of $\mathbb{R}^{n}$. 
Proof. Let $y \in \mathbb{R}^{n}$ be a $W$-general point on the unit sphere $S$ of $V$ where none of the basic invariants of $W$ vanishes. Let $X:=\operatorname{Cone}(W y) \subseteq V$ be the cone over the $W$-orbit of $y$. Let $J$ be any homogeneous ideal with $V_{\mathbb{R}}(J)=X$ and let $\beta$ be the maximum degree of one of its generators. We will construct a homogeneous $W$-invariant form of degree $2 \beta$ which is nonnegative on $\mathcal{H}_{W}$ and which is strictly negative on $y$. To this end, let $h_{1}, \ldots, h_{s}$ be a generating set of the vector space $J_{\beta}$ which is closed under the action of $W$. Define $\phi:=h_{1}^{2}+\cdots+h_{s}^{2}$ and note that $\phi$ is $W$-invariant, nonnegative, and satisfies $V_{\mathbb{R}}(\phi)=X$. Let $\mu=\min \left\{\phi(x): x \in \mathcal{H}_{W} \cap S\right\}$. Then $\mu>0$ and thus $\bar{\phi}:=h_{1}^{2}+\cdots+h_{s}^{2}-\frac{\mu}{2}|x|^{2 \beta}$ is a homogeneous, $W$-invariant form, which is nonnegative on $\mathcal{H}_{W}$ and satisfies $\bar{\phi}(y)=-\frac{\mu}{2}<$ 0 . Next, we obtain an upper bound for the number $\beta$ by constructing homogeneous ideals $J$ with $V_{\mathbb{R}}(J)=X$.

Let $O=\left\{j: 1 \leq j \leq n\right.$ and $\operatorname{deg}\left(\eta_{j}\right)$ is odd $\}$. If $O \neq \emptyset$ then let $\underline{o}:=\min O$ and $\bar{o}=\max O$ and for a basic invariant $\eta_{j}$ define $\overline{\eta_{j}}:=\eta_{j}(y)$. Define homogeneous polynomials $p_{j}$ for $1 \leq j \leq n$ by

$$
p_{j}:=\left\{\begin{array}{l}
\eta_{j}-\overline{\eta_{j}}|x|^{d_{j}}, \text { if } j \notin O \\
\eta_{j} \eta_{\underline{o}}-\overline{\eta_{j} \eta_{\underline{o}}}|x|^{d_{j}+\underline{o}}, \text { if } j \in O
\end{array}\right.
$$

We claim that $V_{\mathbb{R}}\left(p_{1}, \ldots, p_{n}\right)=X$. Equivalently we will show that $V_{\mathbb{R}}\left(p_{1}, \ldots, p_{n}\right) \cap S$ equals $W y \cup-W y$. Since $V_{\mathbb{R}}\left(p_{1}, \ldots, p_{n}\right)$ is generated by homogeneous $W$-invariant forms of even degree it is immediate that $W y \cup-W y \subseteq V_{\mathbb{R}}\left(p_{1}, \ldots, p_{n}\right) \cap S$. For the opposite inclusion, if $x \in V_{\mathbb{R}}\left(p_{1}, \ldots, p_{n}\right) \cap S$ then $\eta_{\underline{o}}(x)^{2}=\eta_{\underline{o}}(y)^{2}$ and thus $\eta_{\underline{o}}(x)= \pm \eta_{\underline{o}}(y)$. It follows that either $\eta_{i}(x)=\eta_{i}(y)$ for all $i$ with $1 \leq i \leq n$ or $\eta_{i}(x)=\eta_{i}(-y)$ for all $i$ with $1 \leq i \leq n$ so that $x \in W y \cup-W y$ (note that the two alternatives coincide if $W$ has no invariants of odd degree). The largest degree of a polynomial $p_{j}$ is $\max \left(d_{n}, \underline{o}+\bar{o}\right)$ and this is an upper bound for $\beta$ in the previous paragraph. As a result, the $W$-invariant form $\bar{\phi}$ obtained from the ideal $J=\left(p_{1}, \ldots, p_{n}\right)$ has degree $2 \max \left(d_{n}, \underline{o}+\bar{o}\right)$, is nonnegative on $\mathcal{H}_{W}$ and is strictly negative on $y$ as claimed.

In the case of irreducible reflection groups the bounds from the previous two theorems can be computed explicitly and we do so in Table 2.1. Theorem 2.14 implies that Theorem 2.13 is sharp whenever the entries of the last two columns of Table 2.1 agree.

For polynomials which are invariant under $A_{n}$, the previous theorems are weaker than Timofte's remarkable half-degree principle. It is a very interesting question whether there is a generalization of the half-degree principle for all finite reflection groups. We conclude by proposing the following conjectural answer, which is a natural generalization of Theorem 2.13.

Conjecture 2.15. Let $j \geq 1$ be an integer. Assume $\|x\|^{2}$ is in the algebra generated by the basic invariants $\eta_{1}, \ldots, \eta_{k}$. If $f$ is a $W$-invariant form of degree $2 d$ with $d_{j-1} \leq d<d_{j}$ then $f$ is nonnegative if and only if $f$ is nonnegative on the $k$-dimensional faces of $\mathcal{H}_{W}$. 
Table 2.1: Degrees of basic invariants of irreducible reflection groups.

\begin{tabular}{|c|l|l|l|}
\hline Root System & Degrees $d_{i}$ & $2 d_{n}$ & $\max \left(2 d_{n}, 2(\underline{o}+\bar{o})\right)$ \\
\hline$A_{n}$ & $2,3, \ldots, n+1$ & $2(n+1)$ & $\left\{\begin{array}{l}2(n+3), n \text { odd } \\
2(n+4), n \text { even. }\end{array}\right.$ \\
\hline$B_{n}$ & $2,4,6, \ldots, 2 n$ & $4 n$ & $4 n$ \\
\hline$D_{n}$ & $2,4,6, \ldots, 2 n-2, n$ & $4 n-4$ & $\left\{\begin{array}{l}4 n, n \text { odd } \\
4 n-4, n \text { even. }\end{array}\right.$ \\
\hline$E_{6}$ & $2,5,6,8,9,12$ & 24 & 28 \\
\hline$E_{7}$ & $2,6,8,10,12,14,18$ & 36 & 36 \\
\hline$E_{8}$ & $2,8,12,14,18,20,24,30$ & 60 & 60 \\
\hline$F_{4}$ & $2,6,8,12$ & 24 & 24 \\
\hline$G_{2}$ & 2,6 & 12 & 12 \\
\hline$H_{3}$ & $2,6,10$ & 20 & 20 \\
\hline$H_{4}$ & $2,12,20,30$ & 60 & 60 \\
\hline$I_{2}(m)$ & $2, m$ & $2 m$ & $\left\{\begin{array}{l}4 m, m \text { odd } \\
2 m, m \text { even. }\end{array}\right.$ \\
\hline
\end{tabular}

Proving Conjecture 2.15 would imply both Timofte's half-degree principle and a generalization of the results of Choi, Lam and Reznick on even symmetric sextics.

\subsection{More Jacobians, Harris Bases, and reducing the di- mension of test sets}

In this section we mainly follow the ideas in [10] and [13]. We show some examples and give some formulas and generalizations. Finally we show how to use Harris bases for finding simpler test sets in some cases.

Definition 2.16. Let $\eta_{1}, \ldots, \eta_{n}$ be basic invariants for $W$ and

$$
B=\left\{\eta_{1}^{a_{11}} \eta_{2}^{a_{12}} \ldots \eta_{n}^{a_{1 n}}, \ldots, \eta_{1}^{a_{m 1}} \eta_{2}^{a_{m 2}} \ldots \eta_{n}^{a_{m n}}\right\}
$$

a basis for the vector space $\mathbb{R}\left[x_{1}, \ldots, x_{n}\right]_{2 d}^{W}$ of $W$-invariant forms of degree $2 d$. We define $J_{W, 2 d}(y)$ as the Jacobian matrix of $B$ with respect to $x_{1}, \ldots, x_{n}$ evaluated at $y$.

Notice that, since the $\eta_{j}$ are algebraically independent, the dimension $m$ of the $\mathbb{R}$ vector space $R_{2 d}^{W}$ is precisely the number of $n$-tuples of nonnegative integers $\left(c_{1}, \ldots, c_{n}\right)$ that 
satisfy

$$
\sum_{j=1}^{n} d_{j} c_{j}=2 d
$$

In other words, the row vectors of $A:=\left(a_{i j}\right)$ in the definition above are precisely the integer points in the simplex

$$
C_{W, 2 d}:=\left\{x \in \mathbb{R}^{n}: d_{1} x_{1}+\ldots+d_{n} x_{n}=2 d, x_{1} \geq 0, \ldots, x_{n} \geq 0\right\}
$$

Example 2.17. A set of basic invariants for $B C_{n}$ can be the power sums $p_{r}(x):=x_{1}^{r}+\ldots+$ $x_{n}^{r}$ for $r=2,4, \ldots, 2 n$. Let's calculate $J_{B C_{3}, 8}(y)$ with respect to the basis $\left\{p_{6} p_{2}, p_{4}^{2}, p_{4} p_{2}^{2}, p_{2}^{4}\right\}$. We just compute the first row and by symmetry the others will follow. For simplicity we denote $p_{r}$ for $p_{r}(x)$ and $\bar{p}_{r}$ for $p_{r}(y)$.

$$
\begin{aligned}
& \frac{\partial p_{6} p_{2}}{\partial x_{1}}(y)=2 y_{1} \bar{p}_{6}+6 y_{1}^{5} \bar{p}_{2}, \quad \frac{\partial p_{4}^{2}}{\partial x_{1}}(y)=8 y_{1}^{3} \bar{p}_{4} \\
& \frac{\partial p_{4} p_{2}^{2}}{\partial x_{1}}(y)=4 y_{1} \bar{p}_{4} \bar{p}_{2}+4 y_{1}^{3} \bar{p}_{2}^{2}, \quad \frac{\partial p_{2}^{4}}{\partial x_{1}}(y)=8 y_{1} \bar{p}_{2}^{3}
\end{aligned}
$$

Thus

$$
J_{B C_{3}, 8}(y)=\left[\begin{array}{cccc}
2 y_{1} \bar{p}_{6}+6 y_{1}^{5} \bar{p}_{2} & 8 y_{1}^{3} \bar{p}_{4} & 4 y_{1} \bar{p}_{2} \bar{p}_{4}+4 y_{1}^{3} \bar{p}_{2}^{2} & 8 y_{1} \bar{p}_{2}^{3} \\
2 y_{2} \bar{p}_{6}+6 y_{2}^{5} \bar{p}_{2} & 8 y_{2}^{3} \bar{p}_{4} & 4 y_{2} \bar{p}_{2} \bar{p}_{4}+4 y_{2}^{3} \bar{p}_{2}^{2} & 8 y_{2} \bar{p}_{2}^{3} \\
2 y_{3} \bar{p}_{6}+6 y_{3}^{5} \bar{p}_{2} & 8 y_{3}^{3} \bar{p}_{4} & 4 y_{3} \bar{p}_{2} \bar{p}_{4}+4 y_{3}^{3} \bar{p}_{2}^{2} & 8 y_{3} \bar{p}_{2}^{3}
\end{array}\right]
$$

or

$$
\left[\begin{array}{lll}
\bar{p}_{6} \nabla p_{2}(y)+\bar{p}_{2} \nabla p_{6}(y) & \left.2 \bar{p}_{4} \nabla p_{4}(y) \quad 2 \bar{p}_{2} \bar{p}_{4} \nabla p_{2}(y)+\bar{p}_{2}^{2} \nabla p_{4}(y) \quad \begin{array}{c}
J_{B C_{3}, 8}(y)= \\
4 \bar{p}_{2}^{3} \nabla p_{2}(y)
\end{array}\right]
\end{array}\right.
$$

Proposition 2.18. Let $\eta_{1}^{a_{11}} \eta_{2}^{a_{12}} \ldots \eta_{n}^{a_{1 n}}, \ldots, \eta_{1}^{a_{n 1}} \eta_{2}^{a_{n 2}} \ldots \eta_{n}^{a_{n n}}$ be $n$ forms and $A=\left(a_{i j}\right)$. Then the Jacobian of these forms is equal to

$$
|A| \eta_{1}^{a_{11}+a_{21}+\ldots+a_{n 1}-1} \ldots \eta_{n}^{a_{1 n}+a_{2 n}+\ldots+a_{n n}-1}\left(\nabla \eta_{1} \wedge \ldots \wedge \nabla \eta_{n}\right)
$$

Proof. We can write the Jacobian as

$$
\begin{gathered}
\left(a_{11} \eta_{1}^{a_{11}-1} \eta_{2}^{a_{12}} \ldots \eta_{n}^{a_{1 n}} \nabla \eta_{1}+\ldots+a_{1 n} \eta_{1}^{a_{11}} \eta_{2}^{a_{12}} \ldots \eta_{n}^{a_{1 n}-1} \nabla \eta_{n}\right) \wedge \\
\left(a_{21} \eta_{1}^{a_{21}-1} \eta_{2}^{a_{22}} \ldots \eta_{n}^{a_{2 n}} \nabla \eta_{1}+\ldots+a_{2 n} \eta_{1}^{a_{21}} \eta_{2}^{a_{22}} \ldots \eta_{n}^{a_{2 n}-1} \nabla \eta_{n}\right) \wedge \\
\vdots \\
\wedge\left(a_{n 1} \eta_{1}^{a_{n 1}-1} \eta_{2}^{a_{n 2}} \ldots \eta_{n}^{a_{n n}} \nabla \eta_{1}+\ldots+a_{n n} \eta_{1}^{a_{n 1}} \eta_{2}^{a_{n 2}} \ldots \eta_{n}^{a_{n n}-1} \nabla \eta_{n}\right)
\end{gathered}
$$


By the multilinearity and alternance of the determinant we can choose one summand from each column so that these have different $\nabla \eta_{i}$ factors and then sum all these determinants. Rearranging the $\nabla \eta_{i}$ in each determinant to index increasing order we factor $\nabla \eta_{1} \wedge \ldots \wedge \nabla \eta_{n}$ and leave the sign of the permutation used for the rearrangement. It remains the product of the $\eta_{j}$ 's, which is the same in each determinant, and the signed products of the $a_{i j}$ whose sum is by definition the determinant of $A$.

As a corollary we have the following lemma, which will be useful to compute Harris bases.

Lemma 2.19. Suppose that the jacobian matrix $J_{W, d}$ has at least as many columns as rows. Take $y \in \mathbb{R}^{n}$ such that no $\eta_{i}$ vanishes at $y$. Then $J_{W, d}(y)$ has full rank if and only if $y$ is a $W$-general point.

Proof. By Propositions 2.18 and 1.20, the jacobian $\nabla \eta_{1} \wedge \ldots \wedge \nabla \eta_{n}$ is not identically zero and in fact vanishes only at $W$-special points. By Proposition 2.18, and the fact that necessarily some $|A|$, corresponding to a maximal minor of $J_{W, d}(y)$, does not vanish, it follows that $J_{W, d}(y)$ has full rank.

If we want to say more about the rank of $J_{W, d}$ we have to look at more minors.

Definition 2.20. Let $\nabla_{I}$ denote the gradient operator with respect to a set of variables indexed by $I$ where $I$ is a subset of $[n]$. For instance, $\nabla_{I} \eta$ is the vector with components $\frac{\partial \eta}{\partial x_{i}}$ where $i$ runs increasingly through the elements of $I$. We write $\left(\begin{array}{c}{[n]} \\ k\end{array}\right)$ for the set of subsets of $[n]$ of size $k$. For example, $\left\{\nabla_{I} \eta_{1} \wedge \cdots \wedge \nabla_{I} \eta_{k}: I \in\left(\begin{array}{c}{[n]} \\ k\end{array}\right)\right\}$ is the set of maximal minors of the matrix $J_{W, k}$.

Proposition 2.21. Let $\eta_{1}, \ldots, \eta_{n}$ be a set of basic invariants for $W$. Let $k \leq n$ and consider the $k$ forms $\eta_{1}^{a_{11}} \eta_{2}^{a_{12}} \ldots \eta_{n}^{a_{1 n}}, \ldots, \eta_{1}^{a_{k 1}} \eta_{2}^{a_{k 2}} \ldots \eta_{n}^{a_{k n}}$ and set $A=\left(a_{i j}\right)$. Then the Jacobian of these forms corresponding to the variables $x_{1}, \ldots, x_{k}$ is equal to

$$
\sum_{\substack{I \subseteq[n] \\|I|=k}}\left|A_{I}\right| \frac{\eta_{1}^{a_{11}+a_{21}+\ldots+a_{k 1}} \ldots \eta_{n}^{a_{1 n}+a_{2 n}+\ldots+a_{k n}}}{\prod_{i \in I} \eta_{i}} \bigwedge_{i \in I} \nabla_{k} \eta_{i}
$$

where the gradients are wedged in index increasing order, $\left|A_{I}\right|$ is the maximal minor of $A$ whose columns are the ones indexed by $I$ and $\nabla_{k}$ denotes the gradient corresponding to the variables $x_{1}, \ldots, x_{k}$.

Proof. We choose a subset $I \subseteq[n]$ with $|I|=k$ and sum all the determinants involving $\bigwedge_{i \in I} \nabla_{k} \eta_{i}$ that appear in the jacobian, following the proof of Proposition 2.18, now sum over all such subsets. 
This last proposition could be used for exhibiting subspaces of forms with low dimensional test sets as in [13, Section 5]. Following their ideas, the following questions arise:

1. When does all the $\left|A_{I}\right|$ have the same sign? For example, find all positroids [23] corresponding to points in the simplex $C_{W, d}$.

2. If $1 \leq i_{1}<\ldots<i_{k} \leq n$, what can be said of

$$
\frac{\nabla_{k} \eta_{i_{1}} \wedge \ldots \wedge \nabla_{k} \eta_{i_{k}}}{\nabla_{k} \eta_{1} \wedge \ldots \wedge \nabla_{k} \eta_{k}} ?
$$

Are these a sort of generalization of Schur functions? And, in view of Proposition 1.10 , are they nonnegative in some fundamental domain of $W$ ?

\subsubsection{Restriction to subspaces}

We show, following [10], that if a psd $W$-invariant form has a zero which is not sufficiently simple, i.e., a $W$-general zero, then it belongs to certain subspace of forms. Let $f$ be a $W$-invariant form of degree $2 d$, then we can write

$$
f=\sum_{\lambda_{i}} c_{\lambda_{i}} \eta_{\lambda_{i}} \text { thus } \frac{\partial f}{\partial x_{i}}=\sum_{\lambda_{i}} c_{\lambda_{i}} \frac{\partial \eta_{\lambda_{i}}}{\partial x_{i}}
$$

where $\eta_{\lambda_{i}}:=\eta_{1}^{\lambda_{i 1}} \ldots \eta_{n}^{\lambda_{i n}}$ for $\lambda_{i}:=\left(\lambda_{i 1}, \ldots, \lambda_{i n}\right)$ and the sum runs through all $\lambda_{i} \in \mathbb{Z}_{\geq 0}^{n}$ such that $\sum_{j=1}^{n} \lambda_{i j} d_{j}=2 d$. Therefore

$$
J_{W, d}\left[\begin{array}{c}
c_{\lambda_{1}} \\
\vdots \\
c_{\lambda_{k}}
\end{array}\right]=\left[\begin{array}{c}
\frac{\partial f}{\partial x_{1}} \\
\vdots \\
\frac{\partial f}{\partial x_{n}}
\end{array}\right]
$$

where the $c_{\lambda_{i}}$ are the coefficients of $f$ in the basis $B$. Therefore, if we identify polynomials in $\mathbb{R}\left[x_{1}, \ldots, x_{n}\right]_{2 d}^{W}$ with its ordered set of coefficients in the basis $B$, we can say $f$ belongs to the kernel of $J_{W, d}(y)$ for any of its singular points $y$, i.e., $\frac{\partial f}{\partial x_{i}}(y)=0$ for all $i=1, \ldots, n$. In particular, we state the following lemma.

Lemma 2.22. If $f$ is psd and $f(y)=0$ then $f \in \operatorname{ker} J_{W, 2 d}(y)$.

Proof. Since $f$ is psd every zero of $f$ is a minimum, hence a singular point.

Definition 2.23. Let $\eta_{1}, \ldots, \eta_{n}$ be basic invariants for a reflection group $W$ and let $U$ be a subspace of $\mathbb{R}\left[x_{1}, \ldots, x_{n}\right]_{d}^{W}$. A Harris basis for $U$ is a basis for $U$ that vanishes at each point of $\mathcal{C}_{y}^{r}$ (assuming Condition 1 holds) for some $r \in\{1, \ldots, n\}$ and some $y \in \mathbb{R}^{n}$. 
Theorem 2.24. If ker $J_{W, d}(v)$ has a Harris basis for every $W$-general point $v$, then $\mathcal{H}_{W}$ is a test set for $R_{2 d}^{W}$.

Proof. Let $f \in R_{2 d}^{W}$ be nonnegative on $\mathcal{H}_{W}$ and suppose $f$ is not psd. Then there exist a $W$ general point $y \in S^{n-1}$ such that $f(y)=\mu<0$ for $\mu:=\min \left\{f(x): x \in S^{n-1}\right\}$. Therefore the form $g(x):=f(x)-\mu\|x\|^{2 d}$ is psd and $g(y)=0$. Since $y$ is $W$-general, Lemma 2.22 implies that $g$ belongs to ker $J_{W, 2 d}(y)$ and therefore $g(x)=\lambda_{1} \nu_{1, y}(x)+\ldots+\lambda_{k} \nu_{k, y}(x)$ where $\left\{\nu_{1, y}, \ldots, \nu_{k, y}\right\}$ is a Harris basis for ker $J_{W, 2 d}(y)$. Now, by Lemma 2.5, there exists a $W$-special point $z$, which we take in $S^{n-1}$ (since a Harris basis "satisfies" Condition 1), in which this basis vanishes, therefore $g(z)=f(z)-\mu=0$, thus $f(z)=\mu<0$, a contradiction.

Remark. The motivation for the definition of a Harris basis comes from the nature of the bases that William Harris found for the kernels of the matrices $J_{B C_{n}, 2 d}$ for some particular values of $n$ and $d$, and were given at [10]. For example, a basis of ker $J_{B C_{3}, 8}(y)$ that appears in [10] is the square of the form:

$$
h(x, y)=p_{2}^{2}(y) p_{4}(x)-p_{4}(y) p_{2}^{2}(x)
$$

This is a Harris basis for every $W$-general point $y$ because:

1. It is a basis for ker $J_{B C_{3}, 8}(\mathrm{y})$ : it is singular at $y$ (and therefore belongs to ker $J_{B C_{3}, 8}(y)$ ), and by Lemma 2.19 ker $J_{B C_{3}, 8}(y)$ is 1-dimensional.

2. It vanishes for every $x \in \mathcal{C}_{y}^{3}$ (the missing invariant is the third one: $p_{6}$ ).

Another example in [10] is a basis for ker $J_{B C_{3}, 10}(y)$ :

$$
\begin{aligned}
& r_{1, y}(x)=h^{2}(x, y) p_{2}(x) \\
& r_{2, y}(x)=h(x, y)\left(p_{2}(y) p_{4}(y) p_{6}(x)-p_{6}(y) p_{2}(x) p_{4}(x)\right)
\end{aligned}
$$

We can check this is a Harris basis that vanishes at $\mathcal{C}_{y}^{3}\left(p_{6}\right.$ appears in $r_{2, y}$ but $r_{2, y}$ is a multiple of $h(x, y)$, which vanishes at $\left.\mathcal{C}_{y}^{3}\right)$. So, if we can construct bases for ker $J_{W, 2 d}$ with the property that each element is a product of at least two (in order to have a singularity at $y$ ) polynomials of the form $\eta_{\lambda}(y) \eta_{\mu}(x)-\eta_{\mu}(y) \eta_{\lambda}(x)$ where $\mu$ and $\lambda$ are compositions such that $\operatorname{deg} \eta_{\mu}=\operatorname{deg} \eta_{\lambda}$, such that at least one invariant is missing (missing in the whole basis or every time in a multiple of others where it is missing) then this will be a Harris basis, and by Theorem 2.24, $\mathcal{H}_{W}$ will be a test set for $R_{2 d}^{W}$.

Harris bases become more interesting when we note that Theorem 2.24 can be combined with Theorem 2.12. For instance, if $J_{W, 2 d}(y)$ admits a Harris basis which depends only on the first $k$ basic invariants, then $k$-dimensional faces of $\mathcal{H}_{W}$ are a test set for forms in $\operatorname{ker} J_{W, 2 d}(y)$. 
Theorem 2.25. If $J_{W, 2 d}(y)$ admits a Harris basis which vanish at $\mathcal{Z}_{y}^{k}$, then $k$-dimensional faces of $\mathcal{H}_{W}$ are a test set for forms in $\operatorname{ker} J_{W, 2 d}(y)$.

Finally, we use Harris bases to derive better test sets for $F_{4}$-invariant forms, of degrees 12 and 14. We first do it without making use of Theorem 2.12 .

\section{An application for $F_{4}$}

First we prove some connexity lemmas that will be helpful when restricting to a subset of $\mathcal{H}_{W} \cap S^{n-1}$ for checking nonnegativity.

Lemma 2.26. Let $\mathcal{H}$ be the union of a set of hyperplanes that pass through the origin in $\mathbb{R}^{n}$ for $n \geq 3$. Then the intersection $\mathcal{H} \cap S^{n-1}$ of $\mathcal{H}$ with the sphere $S^{n-1}$ is connected.

Proof. The intersection of an hyperplane through the origin with the sphere $S^{n-1}$ is a copy of $S^{n-2}$, which is connected for $n \geq 3$ (the only disconnected sphere is $S^{0}$ ). The intersection of any two hyperplanes of $\mathcal{H}$ contains an $(n-2)$-dimensional subspace, and since $n \geq 3$ they intersect in $S^{n-1}$. The set $\mathcal{H} \cap S^{n-1}$ is connected because it is the union of connected sets such that any pair have a point in common.

Definition 2.27. Let $\Phi$ be a root system of rank $n$ and $W$ its associated reflection group, we define $\mathcal{H}_{W}$ to be the union $\bigcup_{\alpha \in \Phi} H_{\alpha}$ of the hyperplanes corresponding to the root system $\Phi$.

Corollary 2.28. If $W$ is a reflection group of rank $n \geq 3$ then $\mathcal{H}_{W} \cap S^{n-1}$ is connected.

$F_{4}$ has degrees $2,6,8,12$. Let $\eta_{2}, \eta_{6}, \eta_{8}, \eta_{12}$ be basic invariants of degree $2,6,8,12$ respectively. The unique degree $d$ such that the number of columns of the matrix $J_{F_{4}, d}$ is one more than the number of variables is 12 : the set $\left\{\eta_{12}, \eta_{8} \eta_{2}^{2}, \eta_{6}^{2}, \eta_{6} \eta_{2}^{3}, \eta_{2}^{6}\right\}$ is a basis for the vector space of $F_{4}$-invariant forms of degree 12. By Lemma 2.19, the kernel of $J_{F_{4}, 12}(y)$, for $y$ an $F_{4}$-general point, is one-dimensional, and in fact it is generated by the square of

$$
T_{12, y}:=\bar{\eta}_{2}^{3} \eta_{6}-\bar{\eta}_{6} \eta_{2}^{3}
$$

A simple system of $F_{4}$ can be $\left\{\alpha_{1}:=\frac{1}{2}\left(e_{1}-e_{2}-e_{3}-e_{4}\right), \alpha_{2}:=e_{2}-e_{3}, \alpha_{3}:=\right.$ $\left.e_{3}-e_{4}, \alpha_{4}:=e_{4}\right\}$ [12, Section 2.10]. Suppose $s_{\alpha_{1}}, s_{\alpha_{2}}, s_{\alpha_{3}}, s_{\alpha_{4}}$ are the reflections across the hyperplanes orthogonal to these roots. We can compute matrix representations of these reflections with respect to the canonical basis of $\mathbb{R}^{4}$ : 


$$
\begin{aligned}
& s_{\alpha_{1}}=\left[\begin{array}{rrrr}
\frac{1}{2} & \frac{1}{2} & \frac{1}{2} & \frac{1}{2} \\
\frac{1}{2} & \frac{1}{2} & -\frac{1}{2} & -\frac{1}{2} \\
\frac{1}{2} & -\frac{1}{2} & \frac{1}{2} & -\frac{1}{2} \\
\frac{1}{2} & -\frac{1}{2} & -\frac{1}{2} & \frac{1}{2}
\end{array}\right], s_{\alpha_{2}}=\left[\begin{array}{cccc}
1 & 0 & 0 & 0 \\
0 & 0 & 1 & 0 \\
0 & 1 & 0 & 0 \\
0 & 0 & 0 & 1
\end{array}\right] \\
& s_{\alpha_{3}}=\left[\begin{array}{llll}
1 & 0 & 0 & 0 \\
0 & 1 & 0 & 0 \\
0 & 0 & 0 & 1 \\
0 & 0 & 1 & 0
\end{array}\right], s_{\alpha_{4}}=\left[\begin{array}{cccc}
1 & 0 & 0 & 0 \\
0 & 1 & 0 & 0 \\
0 & 0 & 1 & 0 \\
0 & 0 & 0 & -1
\end{array}\right]
\end{aligned}
$$

Now we give explicit basic invariants for $F_{4}$; for the following we will only need the first two. A construction of basic invariants for the irreducible Weyl groups can be found at [19]. With the above matrix representations it's easy to verify, that the polynomials

$$
\eta_{2}(x)=x_{1}^{2}+x_{2}^{2}+x_{3}^{2}+x_{4}^{2} \text { and } \eta_{6}(x)=\sum_{i=1}^{4} x_{i}^{6}+5 \sum_{i \neq j} x_{i}^{4} x_{j}^{2}
$$

are $F_{4}$-invariant $\left(|x|^{2}\right.$ is always $W$-invariant, and $\eta_{6}$ is even symmetric so we only need to check that $\eta_{6}$ is invariant under $\left.s_{\alpha_{1}}\right)$. Now if $y$ is any point such that $\eta_{2}(y)=1$, can we find a point $x \in \mathcal{H}_{F_{4}}$ such that $\eta_{2}(x)=1$ and $\eta_{6}(x)=\eta_{6}(y)$ ?

Note we can rewrite

$$
\begin{aligned}
\eta_{6}(x) & =\sum_{i=1}^{4} x_{i}^{6}+5 \sum_{i \neq j} x_{i}^{4} x_{j}^{2} \\
& =\sum_{i=1}^{4} x_{i}^{6}+5 \sum_{i=1}^{4} x_{i}^{4}\left(\eta_{2}(x)-x_{i}^{2}\right)
\end{aligned}
$$

Given that $\eta_{2}(x)=1$ we get

$$
\begin{aligned}
\eta_{6}(x) & =\sum_{i=1}^{4} x_{i}^{6}+5 \sum_{i=1}^{4} x_{i}^{4}\left(1-x_{i}^{2}\right) \\
& =\sum_{i=1}^{4} x_{i}^{6}+5 \sum_{i=1}^{4} x_{i}^{4}-x_{i}^{6} \\
& =5 p_{4}(x)-4 p_{6}(x)
\end{aligned}
$$


Using Lagrange multipliers we get that the critical points of the function $f(x):=5 M_{4}-$ $4 M_{6}$ satisfy $\nabla f(x)=\lambda \nabla \eta_{2}(x)$. Then $10 x_{i}^{3}-12 x_{i}^{5}=\lambda x_{i}$ for $i=1,2,3,4$. So either $x_{i}=0$ or $12 x_{i}^{4}-10 x_{i}^{2}+\lambda=0$. In the latter case we get $x_{i}^{2}=\frac{5 \pm \sqrt{25-12 \lambda}}{12}$ and using that $\eta_{2}(x)=1$ we get one of these cases: $\frac{20}{12}=1, \frac{20+2 \sqrt{25-12 \lambda}}{12}=1, \frac{20+4 \sqrt{25-12 \lambda}}{12}=1, \frac{20-4 \sqrt{25-12 \lambda}}{12}=1$ or $\frac{20-2 \sqrt{25-12 \lambda}}{12}=1$. For real solutions only the last two are possible, which gives $\lambda=\frac{21}{12}$ and $\lambda=\frac{3}{4}$ respectively. In this first case we get $x_{1}^{2}=x_{2}^{2}=x_{3}^{2}=x_{4}^{2}=\frac{1}{4}$, in the second the point $\left(x_{1}^{2}, x_{2}^{2}, x_{3}^{2}, x_{4}^{2}\right)$ must be a permutation of $\left(\frac{1}{12}, \frac{1}{12}, \frac{1}{12}, \frac{3}{4}\right)$, which gives $f=1$ and $f=\frac{11}{9}$ respectively. Now, if some of the $x_{i}=0$ we have four cases: all of the $x_{i}=0$, and then there are no real solutions since $\eta_{2}(x)=1$; exactly three of them are 0 , and then the other one is 1 so $f=1$; exactly two of them are 0 , and then the other two must equal $\pm \frac{1}{\sqrt{2}}$ so $f=\frac{3}{2}$; exactly one of them is 0 , and then the points $\left(x_{1}^{2}, x_{2}^{2}, x_{3}^{2}, x_{4}^{2}\right)$ are permutations of $\left(\frac{1}{3}, \frac{1}{3}, \frac{1}{3}, 0\right)$ or $\left(\frac{1}{6}, \frac{1}{6}, \frac{2}{3}, 0\right)$ and $f=\frac{11}{9}$ or $f=\frac{23}{18}$. Therefore, the following inequality must be true for all $x \in S^{3}$

$$
1 \leq 5 p_{4}(x)-4 p_{6}(x) \leq \frac{3}{2}
$$

Moreover, since the points $(1,0,0,0)$ and $\left(\frac{1}{\sqrt{2}}, \frac{1}{\sqrt{2}}, 0,0\right)$, giving the extremes of this inequality, belong to $H_{\alpha_{3}} \cap H_{\alpha_{4}} \cap S^{3}$, all values in this range can be obtained there because of its connexity (Lemma 2.26) and the continuity of $f$.

The above reasoning proves the following two statements.

Lemma 2.29. For all $y \in S^{3}$ there exists $z \in H_{\alpha_{3}} \cap H_{\alpha_{4}} \cap S^{3}$ such that $T_{12, y}(z)=0$.

Proposition 2.30. Let $f=a \eta_{6}+b \eta_{2}^{3}$ be an $F_{4}$-invariant sextic. Then $f$ is psd if and only if the following equivalent conditions are satisfied

1. $a+b \geq 0$ and $3 a+2 b \geq 0$.

2. $f(1,0,0,0) \geq 0$ and $f(1,1,0,0) \geq 0$.

Proof. Assuming $\eta_{2}=1$ we have $1 \leq \eta_{6} \leq \frac{3}{2}$. Therefore, all values of $f$ in $S^{3}$ range between $f(1,0,0,0)=a+b$ and $\frac{1}{\sqrt{2}^{6}} f(1,1,0,0)=\frac{3}{2} a+b$, and it attains them all. Thus, $f$ is psd if and only if both values are nonnegative.

Proposition 2.31. The set $H_{\alpha_{3}} \cap H_{\alpha_{4}} \cap S^{3}$ is a test set for $F_{4}$-invariant forms of degree 12 .

Proof. Lemma 2.29 implies there exists $z \in H_{\alpha_{3}} \cap H_{\alpha_{4}} \cap S^{3}$ such that $T_{12, y}(z)=0$, therefore $T_{12, y}^{2}$ is a Harris Basis for $J_{F_{4}, 12}$, and by the same proof of Theorem 2.24 we conclude that $H_{\alpha_{3}} \cap H_{\alpha_{4}} \cap S^{3}$ is a test set. 
If $y$ is an $F_{4}$-general point then $J_{F_{4}, 14}(y)$ has dimension 2 and it is generated by

$$
\begin{aligned}
& r_{1, y}:=T_{12, y}^{2} \eta_{2} \\
& r_{2, y}:=T_{12, y}\left(\bar{\eta}_{2} \bar{\eta}_{6} \eta_{8}-\bar{\eta}_{8} \eta_{2} \eta_{6}\right)
\end{aligned}
$$

We only need to check both are linearly independent

$$
r_{1, y}=\bar{\eta}_{2}^{6} \eta_{2} \eta_{6}^{2}-2 \bar{\eta}_{2}^{3} \bar{\eta}_{6} \eta_{2}^{4} \eta_{6}+\bar{\eta}_{6}^{2} \eta_{2}^{7}
$$

and

$$
r_{2, y}=\bar{\eta}_{2}^{4} \bar{\eta}_{6} \eta_{6} \eta_{8}-\bar{\eta}_{2}^{3} \bar{\eta}_{8} \eta_{2} \eta_{6}^{2}-\bar{\eta}_{2} \bar{\eta}_{6}^{2} \eta_{2}^{3} \eta_{8}+\bar{\eta}_{6} \bar{\eta}_{8} \eta_{2}^{4} \eta_{6}
$$

Since the coefficient of $\eta_{2}^{7}$ in $r_{1, y}$ is $\bar{\eta}_{6}^{2}$ and in $r_{2, y}$ is zero, both functions are linearly independent if $\bar{\eta}_{6} \neq 0$. If $\bar{\eta}_{6}=0$ then $y=0$ because all monomials in $\eta_{6}$ are even and all coefficients are nonnegative, but since $y$ is $F_{4}$-general we cannot have $y=0$.

Proposition 2.32. The set $H_{\alpha_{3}} \cap H_{\alpha_{4}} \cap S^{3}$ is a test set for $F_{4}$-invariant forms of degree 14 .

Proof. Since both $r_{1, y}$ and $r_{2, y}$ are divisible by $T_{12, y}$, as in the proof of Proposition 2.31. we conclude that they conform a Harris Basis for $J_{F_{4}, 14}$ and therefore $H_{\alpha_{3}} \cap H_{\alpha_{4}} \cap S^{3}$ is a test set.

Finally note that Propositions 2.31 and 2.32 (but not Proposition 2.30) are trivialized by Theorem 2.25 by only observing that the sets $T_{12, y}^{2}$ and $r_{1, y}, r_{2, y}$ are Harris bases that vanish at $\mathcal{Z}_{y}^{2}$.

\subsection{Future Directions}

We say that an ideal is $W$-invariant if it is generated by $W$-invariant polynomials. A $W$-invariant (real) variety is the set of common (real) zeros of a $W$-invariant ideal. Suppose that a $W$-invariant real variety $X$ does not intersect $\mathcal{H}_{W}$, then, as chambers partition $\mathbb{R}^{n} \backslash \mathcal{H}_{W}$ into $|W|$ disjoint connected components, $X$ should have at least $|W|$ connected components.

Problem. Compute the number of real connected components of a real variety $X$ given by some basic invariants for $W$ :

$$
X=\left\{x \in \mathbb{R}^{n}: \eta_{i_{1}}(x)=\eta_{i_{1}}(y), \ldots, \eta_{i_{k}}(x)=\eta_{i_{k}}(y)\right\}
$$

where $1 \leq i_{1}<\ldots<i_{k} \leq n$ and $y \in \mathbb{R}^{n}$.

How does the degrees of a parabolic subgroup relate to the degrees of $W$ ? 


\section{Bibliography}

[1] Gérard P. Barbançon, Whitney regularity of the image of the Chevalley mapping. arXiv:1410.3504.

[2] Grigoriy Blekherman, There are significantly more nonnegative polynomials than sums of squares, Israel J. Math. 153 (2006), 355-380.

[3] Grigoriy Blekherman, Pablo A. Parrilo, and Rekha R. Thomas, Semidefinite optimization and convex algebraic geometry, volume 13 of MOS-SIAM Series on Optimization. Society for Industrial and Applied Mathematics (SIAM), 2012.

[4] M.D. Choi, T.Y. Lam, B. Reznick, Even symmetric sextics. Math. Z. 195, no. 4, 559580, (1987).

[5] C. De Concini, C. Procesi, Topics in hyperplane arrangements, polytopes and boxsplines, Springer 2010.

[6] Eisenbud D.:Commutative Algebra with a view towards algebraic geometry.

[7] M. R. Garey and D. S. Johnson, Computers and Intractability: A guide to the theory of NP-completeness. W. H. Freeman and Company, 1979.

[8] M. Ghasemi and M. Marshall, Lower bounds for polynomials using geometric programming, SIAM J. Optim. 22 (2012), no. 2, 460473.

[9] Karin Gatermann, Pablo Parrilo, Symmetry groups, semidefinite programs, and sums of squares, Journal of Pure and Appl. Algebra, Vol. 192, No. 1-3, pp. 95-128, 2004.

[10] William Harris, Real even symmetric ternary forms, J. Algebra 222 (1999), no. 1, 204-245.

[11] David Hilbert, ber die Darstellung definiter Formen als Summe von Formenquadraten, Math. Ann. 32 (1888), 342-350; see Ges. Abh. 2, 154-161, Springer, Berlin, 1933, reprinted by Chelsea, New York, 1981. 
[12] James E. Humphreys, Reflection Groups and Coxeter Groups, Cambridge University Press, Cambridge, 1990.

[13] Sadik Iliman, Timo De Wolff, Low dimensional test sets for nonnegativity of even symmetric forms. arXiv:1303.4241.

[14] Sadik Iliman, Timo De Wolff, Lower Bounds for Polynomials with Simplex Newton Polytopes Based on Geometric Programming. arXiv preprint arXiv:1402.6185 (2014).

[15] Richard Kane, Reflection Groups and Invariant Theory. Springer, New York (2001).

[16] Kollar J.: Lectures on Resolution of Singularities, Annals of Mathematics Studies Vol. 166, Princeton University Press, 2007.

[17] Jean B. Lasserre, A sum of squares approximation of nonnegative polynomials, SIAM J. Optim. 16, pp. 751-765.

[18] Jean B. Lasserre, Moments, positive polynomials and their applications. Imperial College Press, London, 2009.

[19] C.Y. Lee, Invariant polynomials of Weyl group and applications to the centres of universal enveloping algebras, Can. J. Math, Vol XXVI. No. 3, 1974, pp. 583-592.

[20] Neusel M., Smith L.:Invariant theory of finite groups, Mathematical Surveys and Monographs, 94, American Mathematical Society, 2002.

[21] Pablo A. Parrilo, Semidefinite programming relaxations for semialgebraic problems. Math. Prog., 96 (2, Ser. B): 293-320, 2003.

[22] Pablo A. Parrilo, Bernd Sturmfels, Minimizing polynomial functions, arXiv:math/0103170v1.

[23] Alexander Postnikov, Total positivity, Grassmannians, and networks, arXiv:math/0609764v1.

[24] Claudio Procesi, Positive symmetric functions, Adv. Math., 29: 219-225, 1978.

[25] Cordian Riener, On the degree and half-degree principle for symmetric polynomials, J. Pure Appl. Algebra 216 (2012), no. 4, 850-856.

[26] Cordian Riener, Symmetric semi-algebraic sets and non-negativity of symmetric polynomials, arXiv:1409.0699.

[27] C. Riener, R. Sanyal, Personal Communication.

[28] Richard Stanley, Enumerative Combinatorics, vol 2. 
[29] Robert Steinberg, Invariants of finite reflection groups, Canad. J. Math. 12 (1960), 616-618.

[30] Steurer D., Barak B., Sum-of-Squares proofs and the quest toward optimal algorithms, ICM 2014.

[31] Bernd Sturmfels, Algorithms in Invariant Theory, second ed., Texts and Monographs in Symbolic Computation, SpringerWienNew York, 2008.

[32] V. Timofte, On the positivity of symmetric polynomial functions, I. General results, J. Math. Anal. Appl. 284 (2003), no. 1, 174-190. 